\title{
TOWARDS A THEORY FOR NEPTUNE'S ARC RINGS
}

\author{
Peter Goldreich \\ California Institute of Technology, Pasadena, California 91125 \\ ScotT Tremaine ${ }^{a)}$ \\ Canadian Institute for Theoretical Astrophysics, University of Toronto, Toronto, Ontario M5S 1A1, Canada \\ and \\ Department of Physics, Massachusetts Institute of Technology, Cambridge, Massachusetts 02138
}

NiCOLE BORDERIES

Observatoire du Pic du Midi et de Toulouse, 31400, Toulouse, France

Received 23 April 1986

\begin{abstract}
We propose that the incomplete rings of Neptune consist of a number of short arcs centered on the corotation resonances of a single satellite. The satellite must have a radius of order $100 \mathrm{~km}$ or more and move on an inclined orbit. Corotation resonances are located at potential maxima. Thus, mechanical energy dissipated by interparticle collisions must be continually replenished to prevent the arcs from spreading. We show that each corotation resonance is associated with a nearby Lindblad resonance, which excites the ring particles' orbital eccentricity, thus supplying the energy required to maintain the arc. The ultimate energy reservoir is the satellite's orbital energy. Therefore, interaction with the arcs damps the satellite's orbital inclination. The self-gravity of the arcs limits their contraction and enforces a relation between arc length and mass. The estimated arc masses are so small, of order $10^{16} \mathrm{~g}$, that the satellite's orbital inclination suffers negligible decay over the age of the solar system. The inferred surface mass densities are comparable to those found in the major rings of Saturn and Uranus.
\end{abstract}

\section{INTRODUCTION}

Recent occultation observations have established Neptune as a member of the family of ringed planets. The most striking feature of Neptune's rings is that they do not completely encircle the planet; for example, the occultation event of 22 July 1984 (Hubbard et al. 1986) was observed on immersion but not on emersion, and the event of 20 August 1985 was observed at two telescopes on Mauna Kea (Nicholson 1986) but was not detected at Flagstaff (Wasserman 1986). These data suggest that the occulting material is confined to a number of short, $\leqslant 10^{4} \mathrm{~km}$, arcs.

The dynamical theory discussed in this paper is based on the assumption that Neptune has at least one satellite with semimajor axis $a_{\mathrm{s}} \lesssim 10^{5} \mathrm{~km}$. This satellite may already have been detected: Reitsema et al. (1982) observed an occultation event on 24 May 1981 which could be due to a satellite of radius $R_{\mathrm{s}}>90 \mathrm{~km}$ and $a_{\mathrm{s}}=(7.5 \pm 2.5) \times 10^{4} \mathrm{~km}$. We note that the probability $p$ of the accidental detection of such a satellite in an occultation is small but not negligible: after $N$ occultations (counting immersion and emersion as separate events $), p=N R_{\mathrm{s}} /\left(a_{\mathrm{s}} \sin I_{\mathrm{N}}\right)$, where $I_{\mathrm{N}}=29^{\circ}$ is Neptune's obliquity to the ecliptic. By the end of 1985, at least $N=20$ occultations have been observed and so $p \gtrsim 0.05$.

We adopt the values $a_{\mathrm{s}}=7.5 \times 10^{4} \mathrm{~km}, R_{\mathrm{s}}=150 \mathrm{~km}$, and $i_{\mathrm{s}}=0.1$ for our hypothetical satellite. The values for the semimajor axis and satellite radius are consistent with the occultation evidence and the value for the inclination $i_{\mathrm{s}}$ is a guess we use to set a scale in numerical examples.

The conclusions reached in this paper are based on simple applications of standard celestial mechanics perturbation theory. Only our principal results are reported here. Detailed calculations, together with a more complete set of results, will be presented in a later paper.

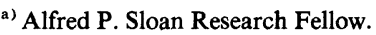

\section{TEST PARTICLE MOTION}

\section{Corotation Resonances}

The presence of arcs strongly implicates satellite corotation resonances as an essential ingredient of the underlying dynamics. The most familiar resonances of this kind are located at the triangular Lagrange points, $L_{4}$ and $L_{5}$, and have pattern speeds equal to the satellite mean motion $n_{\mathrm{s}}$. A satellite that moves on an inclined orbit has additional corotation resonances, the strongest of which have pattern speeds $n_{\mathrm{c}}$ that satisfy

$$
m n_{\mathrm{c}}=(m+1) n_{\mathrm{s}}-\dot{\Omega}_{\mathrm{s}},
$$

where $m$ is any positive or negative integer and $\dot{\Omega}_{\mathrm{s}}$ is the nodal precession rate of the satellite's orbit. We refer to these as the principal corotation resonances. For simplicity, we specialize to corotation resonances which lie inside the satellite orbit (positive $m$ ). Arcs associated with outer corotation resonances are essentially the same as those associated with inner corotation resonances. We shall indicate later why, within our theory, the Lagrange points do not provide plausible sites for ring arcs.

The corotation resonance perturbation potential is

$$
\Phi_{\mathrm{c}}=-i_{\mathrm{s}}^{2} A_{\mathrm{c}} \cos \Psi_{\mathrm{c}},
$$

with coefficient (e.g., Brouwer and Clemence 1961)

$$
A_{\mathrm{c}}=\frac{M_{\mathrm{s}}}{M_{\mathrm{p}}}\left(n_{\mathrm{s}} a_{\mathrm{s}}\right)^{2} \frac{\beta}{8} b_{3 / 2}^{2 m+1}(\beta), \quad m>0,
$$

and critical argument

$$
\Psi_{\mathrm{c}}=2 m\left(\lambda-n_{\mathrm{c}} t\right) \text {. }
$$

Here $\lambda$ is the mean longitude, $t$ is the time, $M_{\mathrm{s}} / M_{\mathrm{p}}$ is the satellite-planet mass ratio, $\beta=a / a_{\mathrm{s}}<1$ is the test particlesatellite semimajor-axis ratio, and $b_{\mathrm{s}}^{i}(\beta)$ is a Laplace coeffi- 
cient. In writing Eqs. (4), we have implicitly set $\lambda_{\mathrm{s}}=\Omega_{\mathrm{s}}$ $=0$ at $t=0$.

Stable equilibrium corresponds to the potential maximum, which lies at $\Psi_{\mathrm{c}}=\pi$ (note $\left.A_{\mathrm{s}}>0\right)$. Small amplitude librations of test particles about the equilibrium point are described by

$$
\Psi_{\mathrm{c}}=\pi+D_{\mathrm{c}} \cos (\alpha t+\delta),
$$

and by $a=a_{\mathrm{c}}+\Delta a$, where $n_{\mathrm{c}}^{2} a_{\mathrm{c}}^{3}=G M_{\mathrm{p}}$ and

$$
\Delta a=\Delta a_{m} \sin (\alpha t+\delta)=\frac{a_{\mathrm{c}} \alpha D_{\mathrm{c}}}{3 m n_{\mathrm{c}}} \sin (\alpha t+\delta) .
$$

Here $0<D_{\mathrm{c}} \ll 1$ and the libration frequency $\alpha>0$ is given by

$$
\alpha^{2}=12 m^{2} i_{\mathrm{s}}^{2} \frac{A_{\mathrm{c}}}{a_{\mathrm{c}}^{2}} \text {. }
$$

The libration trajectory is an ellipse centered on the equilibrium point with semimajor and semiminor axes given by $a_{\mathrm{c}} D_{\mathrm{c}} /(2 m)$ and $\Delta a_{\mathrm{m}}$, respectively. Numerical evaluation of $\Delta a_{m}$ in the limit $m \gg 1$ [see Eq. (15) below] yields

$$
\Delta a_{\mathrm{m}}=9.4 \mathrm{~km} \mathrm{mi} D_{\mathrm{c}}\left(\frac{\rho_{\mathrm{s}}}{\mathrm{g} \mathrm{cm}^{-3}}\right)^{1 / 2}\left(\frac{R_{\mathrm{s}}}{150 \mathrm{~km}}\right)^{3 / 2},
$$

where $\rho_{\mathrm{s}}$ is the satellite density, and we have taken $a_{\mathrm{s}}$ $=7.5 \times 10^{4} \mathrm{~km}$.

The appropriate values of $m$ and $D_{\mathrm{c}}$ are quite uncertain. Most of the reported occultation events are at radii which lie within about $20 \%$ of the location of the hypothetical satellite, which suggests $m \gtrsim 3$. The fractional azimuth subtended by $2 m$ equal libration ellipses with amplitude $D_{\mathrm{c}}$ is $D_{\mathrm{c}} / \pi$; the observed success rate of about 0.1 thus suggests $D_{\mathrm{c}} \approx 0.3$ if all equilibrium sites of just a single $m$ are occupied. However, the appropriate value for $D_{\mathrm{c}}$ would be larger if some equilibrium sites were vacant and smaller if material were present at several values of $m$. In numerical examples we adopt $m=6$ and $D_{\mathrm{c}}=0.3$.

Arcs composed of particles librating about corotation resonances make neat fits to the occultation observations (see Fig. 1). However, the corotation equilibria are at potential

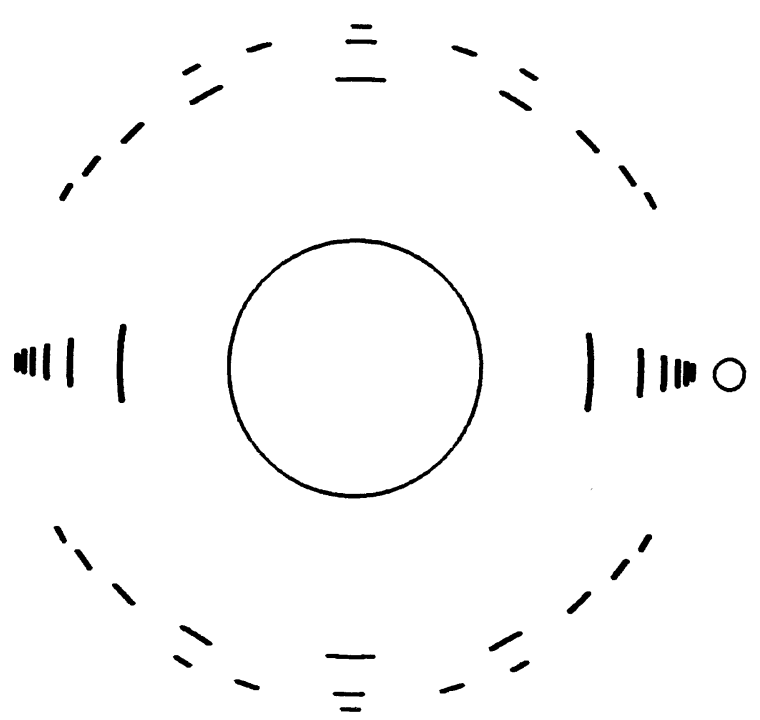

FIG. 1. Schematic picture of ring arcs due to a satellite with semimajor axis $a_{\mathrm{s}}=3 R_{\mathrm{p}}$. Arcs are shown for $m=1, \ldots, 6$ with the satellite at maximum elevation. The corotation libration amplitude is $\pi / 10$, so that $10 \%$ of the azimuth is covered for each $m$. maxima. Thus, mechanical energy dissipated by interparticle collisions must be replenished or the arcs would not remain confined. One possibility, already suggested in a slightly different context by Lissauer (1985), is that a second satellite shepherds each arc. We adopt the more economical hypothesis that the requisite energy is supplied by the Lindblad resonance that is associated with each corotation resonance. Next we examine these Lindblad resonances.

\section{Lindblad Resonances}

The principal Lindblad resonances have pattern speed $n_{\mathrm{s}}$ and are located where

$$
m n_{\mathrm{L}}=(m+1) n_{\mathrm{s}}-\dot{\bar{\omega}}_{\mathrm{L}} .
$$

Here $n_{\mathrm{L}}$ and $\dot{\bar{\omega}}_{\mathrm{L}}$ refer to the test particle; $\dot{\bar{\omega}}_{\mathrm{L}}$ excludes the contribution due to the Lindblad perturbation potential itself.

Comparison of Eqs. (1) and (9) yields

$$
\delta n \equiv n_{\mathrm{c}}-n_{\mathrm{L}}=\frac{\dot{\bar{\omega}}_{\mathrm{L}}-\dot{\Omega}_{\mathrm{s}}}{m}>0,
$$

from which it follows that for $m \gg 1$

$$
\delta a \equiv a_{\mathrm{L}}-a_{\mathrm{c}}=\frac{2}{3} a \frac{\delta n}{n}=2 J_{2}\left(\frac{R_{\mathrm{p}}}{a}\right)^{2} \frac{a}{m},
$$

where $R_{\mathrm{p}}$ is the planetary radius and $J_{2}$ is the dynamical oblateness. Substituting appropriate numerical values (Harris 1984), $J_{2}=0.0043, R_{\mathrm{p}}=2.523 \times 10^{4} \mathrm{~km}$, and $a_{\mathrm{s}}$ $=7.5 \times 10^{4} \mathrm{~km}$, we obtain $\delta a=73 \mathrm{~m}^{-1} \mathrm{~km}$. by

The Lindblad-resonance perturbation potential is given

$$
\Phi_{\mathrm{L}}=e A_{\mathrm{L}} \cos \Psi_{\mathrm{L}},
$$

with coefficient

$$
A_{\mathrm{L}}=\frac{M_{\mathrm{s}}}{M_{\mathrm{p}}}\left(n_{\mathrm{s}} a_{\mathrm{s}}\right)^{2}\left(m+1+\frac{\beta}{2} \frac{d}{d \beta}\right) b_{1 / 2}^{m+1}(\beta), \quad m>0
$$

and critical argument

$$
\Psi_{\mathrm{L}}=m \lambda+\bar{\omega}-(m+1) n_{\mathrm{s}} t .
$$

In the limit $m \gg 1$ (Borderies et al. 1984),

$$
\begin{aligned}
& A_{\mathrm{c}}=\frac{3 m^{2}}{4 \pi} \frac{M_{\mathrm{s}}}{M_{\mathrm{p}}}\left(n_{\mathrm{s}} a_{\mathrm{s}}\right)^{2} K_{1}(4 / 3), \\
& A_{\mathrm{L}}=\frac{m}{\pi} \frac{M_{\mathrm{s}}}{M_{\mathrm{p}}}\left(n_{\mathrm{s}} a_{\mathrm{s}}\right)^{2}\left[2 K_{0}(2 / 3)+K_{1}(2 / 3)\right]
\end{aligned}
$$

Here $K_{0}$ and $K_{1}$ denote modified Bessel functions. We define the shorthand notation $f \equiv K_{1}(4 / 3)=0.354$ and $g \equiv 2 K_{0}(2 / 3)+K_{1}(2 / 3)=2.52$.

The Lindblad potential $\Phi_{\mathrm{L}}$ does not significantly affect the perturbations of $\Psi_{\mathrm{c}}$ and $\Delta a$ given by Eqs. (5) and (6). However, stable equilibrium implies $\Psi_{\mathrm{L}}=0$, eccentricity

$$
e_{0}=\frac{A_{\mathrm{L}}}{m a_{\mathrm{c}}^{2} n_{\mathrm{c}} \delta n},
$$

and apsidal line orientation

$$
\bar{\omega}=\Omega_{\mathrm{s}} \pm \frac{\pi}{2} \text {. }
$$

The $2 m$ equilibrium points alternate between these two ellipses and are equally spaced in azimuth (see Figs. 1 and 2). 
Acting with the corotation potential, the Lindblad potential produces periodic perturbations

$$
\begin{aligned}
& \Psi_{\mathrm{L}}=D_{\mathrm{L}} \cos (\alpha t+\delta), \\
& \Delta e=-\frac{g \alpha}{9 f m^{3} i_{\mathrm{s}}^{2} n} D_{\mathrm{L}} \sin (\alpha t+\delta), \\
& \Delta \bar{\omega}=\left(\frac{2 D_{\mathrm{L}}-D_{\mathrm{c}}}{2}\right) \cos (\alpha t+\delta),
\end{aligned}
$$

where

$$
D_{\mathrm{L}}=\frac{\alpha^{2}}{2\left[\alpha^{2}-(m \delta n)^{2}\right]} D_{\mathrm{c}} \text {. }
$$

There are two ways to view the motion of a test particle in the combined corotation and Lindblad potentials. One, which we have just described, is to consider the Lindblad resonance as perturbing the libration about corotation. Alternately (see Fig. 2), we may picture a collection of test particles confined within a corotation ellipse as filling a limited longitude $D_{\mathrm{c}} / m$ of a Keplerian elliptic ring, which has mean eccentricity $e_{0}$ and dimensionless eccentricity gradient

$$
q=a \frac{\Delta e}{\Delta a}=-\frac{g}{3 f m^{2} i_{\mathrm{s}}^{2}} \frac{D_{\mathrm{L}}}{D_{\mathrm{c}}}
$$

The particles travel around the Keplerian ellipse at the fast angular frequency $n_{\mathrm{c}}$ and circulate about the corotation points at the slow angular frequency $\alpha$. This view is particularly convenient when we come to discuss the stresses due to self-gravity and particle collisions because it enables us to make contact with our previous work on narrow elliptic rings.

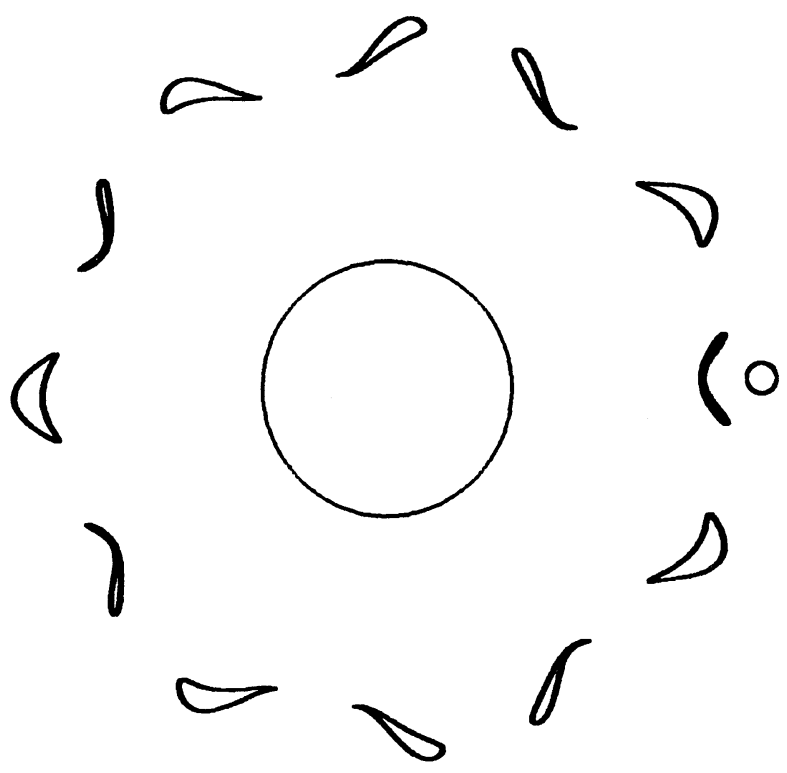

FIG. 2. Enlarged picture of the geometry of ring arcs with $m=6$ due to a satellite with $i_{\mathrm{s}}=0.1, \rho_{\mathrm{s}}=1 \mathrm{~g} \mathrm{~cm}^{-3}, R_{\mathrm{s}}=150 \mathrm{~km}, a_{\mathrm{s}}=7.5 \times 10^{4} \mathrm{~km}$. The satellite is at maximum elevation. Radial variations relative to the resonant semimajor axis, $a_{\mathrm{c}}=6.77 \times 10^{4} \mathrm{~km}$, have been enlarged by a factor 200. The corotation libration amplitude is $D_{\mathrm{c}}=\pi / 2$. Other parameters are $e_{0}=0.00033,2 \Delta a_{m}=15.2 \mathrm{~km}$, and $q=0.85$.

\section{COLLECTIVE EFFECTS}

\section{Self-Gravity}

We adopt a crude expression for the apsidal precession rate due to self-gravity in a narrow elliptic ring (Borderies $e t$ al. 1983, 1985),

$$
\dot{\bar{\omega}}_{\mathrm{sg}}=\frac{n \Sigma a^{2}}{M_{\mathrm{p}} e} q \sin (\alpha t+\delta),
$$

where $\Sigma$ is the surface density. Equation (24) is based on a two-streamline approximation to the ring and holds in the limit of small $q$. The $\sin (\alpha t+\delta)$ factor has been added to account for the oscillation in the sign of $\dot{\omega}_{\mathrm{sg}}$ as the particle librates between the inside and outside of the arc.

The addition of self-gravity modifies the relation between $D_{\mathrm{L}}$ and $D_{\mathrm{c}}$, given for test-particle orbits by Eq. (22) so that it now reads

$$
\begin{aligned}
& {\left[2-\frac{2 \pi}{f m^{4} i_{\mathrm{s}}^{2}} \frac{M_{\mathrm{p}}}{M_{\mathrm{s}}} J_{2}^{2}\left(\frac{R_{\mathrm{p}}}{a}\right)^{4}\right.} \\
& \left.\quad-\frac{4 \pi}{3 f^{2} m^{5} i_{\mathrm{s}}^{4}}\left(\frac{M_{\mathrm{p}} M_{\mathrm{r}}}{M_{\mathrm{s}}^{2}}\right) J_{2}\left(\frac{R_{\mathrm{p}}}{a}\right)^{2} \frac{1}{D_{\mathrm{c}}^{3}}\right] D_{\mathrm{L}}=D_{\mathrm{c}} .
\end{aligned}
$$

In writing Eq. (25) we have eliminated the surface density in terms of the total mass of the arc $M_{\mathrm{r}}=\pi a D_{\mathrm{c}} \Delta a_{\mathrm{m}} \Sigma /(2 \mathrm{~m})$ and also substituted the expressions given in Eqs. (7), (11), and (17) for $\alpha, \delta n$, and $e_{0}$.

Inserting numerical values into Eq. (25), we arrive at

$$
\left(\frac{43}{m^{4} i_{\mathrm{s}}^{2}}\right)\left(\frac{10^{22} \mathrm{~g}}{M_{\mathrm{s}}}\right)\left(\frac{7.5 \times 10^{4} \mathrm{~km}}{a}\right)^{4},
$$

and

$$
\left(\frac{0.17}{m^{5} l_{\mathrm{s}}^{4} D_{\mathrm{c}}^{3}}\right)\left(\frac{M_{\mathrm{r}}}{10^{16} \mathrm{~g}}\right)\left(\frac{10^{22} \mathrm{~g}}{M_{\mathrm{s}}}\right)^{2}\left(\frac{7.5 \times 10^{4} \mathrm{~km}}{a}\right)^{2},
$$

for the magnitudes of the second and third terms on the lefthand side of the equation. For our nominal values $m=6, i_{\mathrm{s}}$ $=0.1, R_{\mathrm{s}}=150 \mathrm{~km}, \rho_{\mathrm{s}}=1 \mathrm{~g} \mathrm{~cm}^{-3}, D_{\mathrm{c}}=0.3, a \approx a_{\mathrm{s}}$ $=7.5 \times 10^{4} \mathrm{~km}$, the two terms have values of order 2.3 and $3.8\left(M_{\mathrm{r}} / 10^{16} \mathrm{~g}\right)$. For this particular set of parameters, $\left|D_{\mathrm{L}} / D_{\mathrm{c}}\right|$ is close to 1 for small $M_{\mathrm{r}}$ and is proportional to $M_{\mathrm{r}}^{-1}$ for $M_{\mathrm{r}} \gg 10^{16} \mathrm{~g}$.

\section{Viscous Stresses}

Consider an arc filled with small particles. It is straightforward to show that the dominant terms in the variations of surface density and rate of strain are identical to those we found previously for narrow, eccentric, Keplerian rings (Borderies et al. 1982, 1983). Both quantities are determined by the parameter $q$ defined in Eq. (23). Thus we may apply relations derived in earlier papers to determine the viscous evolution of Neptune's arcs. We note that $q$ is constant throughout the corotation ellipse, so long as viscous stresses are small and self-gravity is either absent or adequately described by our two-streamline approximation [ $\mathrm{cf}$. Eqs. (23) and (25)].

As before, the viscous luminosity of angular momentum $L_{H}^{\mathrm{v}}$ plays a crucial role. $L_{H}^{\mathrm{v}}$ is the rate at which angular momentum crosses the streamline with semimajor axis $a$. Its precise value depends upon $|q|, \Sigma$, and the details of the kinetic model of particle collisions. All that concerns us here is the qualitative nature of the $|q|$ dependence of $L_{H}^{\mathrm{V}}$, which is common to all cases. In particular, $L_{H}^{\mathrm{v}}$ is positive for 
$|q| \ll 1$ and negative for $1-|q| \ll 1$; the exact value $q_{\mathrm{c}}$ at which $L_{\mathrm{H}}^{\mathrm{v}}$ changes sign is model dependent. The sign change is related to the presence, for $|q|>3 / 4$, of an interval of longitude that is centered at the point where the surface density reaches its maximum value and within which the radial component of the angular-velocity gradient is positive.

To evaluate the effect of the viscous stress on the evolution of $D_{c}$, we apply the method of variation of constants to Eqs. (5) and (6) and obtain

$$
\frac{d D_{\mathrm{c}}^{2}}{d t}=-\frac{3 n_{\mathrm{c}}}{\pi i_{\mathrm{s}}^{2} A_{\mathrm{c}} a_{\mathrm{c}}^{2} \Sigma}\left\langle\frac{\partial L_{H}^{\mathrm{v}}}{\partial a} \Delta a\right\rangle,
$$

where $\langle\cdot\rangle$ denotes a time average over one libration. If the ring arc has a sharp outer boundary, then $\left\langle\left(L_{H}^{\mathrm{v}} / \Sigma\right) \Delta a\right\rangle$ must vanish there. Thus, $|q|=q_{\mathrm{c}}$ must be satisfied on the outer boundary. For interior particles the situation is more complicated since the viscous contribution to the evolution of $D_{\mathrm{c}}$ may be balanced by the contributions due to the satellite and to the arc's self-gravity. More detailed, model dependent calculations are required to determine the variations of $\Sigma$ and $q$ as functions of $D_{c}$; however, the principal feature of these calculations can be anticipated by a simple heuristic argument. Consider a given azimuth, say, $\Psi_{c}=\pi$ (the minor axis of the libration ellipse). Since $L_{H}^{\mathrm{v}}=0$ at the boundaries $\Delta a= \pm \Delta a_{\mathrm{m}}$, the simplest possible spatial variation is $L_{H}^{\mathrm{v}}(\Delta a)=K\left(\Delta a_{\mathrm{m}}^{2}-\Delta a^{2}\right)$. Thus $K>0$ implies $L_{H}^{\mathrm{v}}>0$ and $|q|<q_{\mathrm{c}}$, while $K<0$ implies $|q|>q_{\mathrm{c}}$. If the spatial variation of $L_{H}^{\mathrm{v}}$ is similar at other azimuths, then $\left\langle\left(\partial L_{H}^{\mathrm{v}} / \partial a\right) \Delta a\right\rangle$ is negative when $K$ is positive and vice versa. Hence we conclude that $D_{\mathrm{c}}^{2}$ decreases with time (i.e., the ring arc contracts) if and only if $|q|>q_{\mathrm{c}}$.

\section{DISCUSSION}

\section{Arc Contraction}

The condition that $L_{H}^{\mathrm{v}}=0$ or $|q|=q_{\mathrm{c}}$ at the outer boundary of an arc determines, through Eqs. (23) and (25), a relation between the length of the arc $a D_{c} / m$ and its mass $M_{\mathrm{r}}$. If the arc length were much larger than the equilibrium value, the effects of self-gravity on $q$ would be small. Let us assume that, in this case, Eqs. (23) and (25) predict that $|q|>q_{\mathrm{c}}$. The considerations of the previous subsection then imply that the arc would contract. The contraction of the arc would increase the importance of self-gravity. If the initial value of $q$ were positive, $q$ would decrease monotonically until $q=q_{\mathrm{c}}$. If $q$ were initially negative, it would first decrease through more negative values, then change sign and subsequently decrease until $q=q_{\mathrm{c}}$. In either case, when $q=q_{\mathrm{c}}$ the ring would have reached the equilibrium state consistent with its mass.

The preceding discussion indicates why a diffuse arc could contract. It makes apparent that a necessary condition for the existence of arcs is that $|q|>q_{\mathrm{c}}$ in the absence of selfgravity. It does not address the more difficult issue of how material is initially captured into libration about corotation.

\section{Source Material}

There are two obvious sources for the material in arcs, one external and the other internal.

Small particles, which slowly spiral either toward or away from Neptune under the influence of radiation or plasma drag, might be captured into libration about a corotation resonance. The analysis of the capture dynamics is outside the scope of this paper, as it requires treatment of particle motion near a separatrix and our analysis has been restricted to small oscillations about equilibrium. Nevertheless, it is clear that this external source would lead to similar amounts of material in all of the arcs associated with a given $m$.

Mass lost at low speeds, perhaps as the result of impacts, from a small satellite trapped at the corotation resonance could populate the libration orbits. The satellite might provide the dominant contribution to the self-gravity of the arc and thus render invalid the mass estimate given by Eq. (25). Of course, if internal sources are required, we expect very few potential arcs to be filled.

\section{Sites For Arcs}

The requirement that $|q|>q_{\mathrm{c}}$ in the absence of self-gravity severely constrains the values of $m$ at which arcs can form. For our standard satellite parameters, the allowed values of $m$ are 5-11.

Arcs might also be found around higher-order corotation resonances for which $n_{\mathrm{c}} / n_{\mathrm{s}} \approx(m+j) / m$, with $|j|>1$. The strengths of these resonances may be assessed by noting that $m i_{\mathrm{s}}$ is the appropriate small parameter in the expansion of the perturbation potential.

\section{The Lagrange Points}

The triangular Lagrange points, $L_{4}$ and $L_{5}$, might be considered very promising locations for arcs because they are the sites of the strongest, dynamically stable, corotation resonances. Furthermore, these resonances do not require $i_{\mathrm{s}}>0$ and undoubtedly are stable so long as $i_{\mathrm{s}} \ll 1$. Indeed, Lissauer (1985) has proposed that the Neptune ring arcs consist of material librating about the triangular points of one satellite which is shepherded by a second satellite.

It is remarkable that our single-satellite hypothesis excludes the triangular points as suitable locations for arcs because they are not associated with Lindblad resonances. Thus small particles tend to diffuse away from the triangular points rather than accumulate around them.

\section{Effect Of Arcs On Satellite's Orbit}

Our estimated arc mass is much smaller than the satellite mass. This implies that the arcs cannot store a significant fraction of the total energy and angular momentum of the combined arcs-satellite system. Thus, the satellite's orbital energy diminishes at the same rate that energy is dissipated by inelastic collisions in the arcs, while the component of the satellite's orbital angular momentum that is normal to the planet's equatorial plane remains constant. It follows that $a_{\mathrm{s}}$ and $i_{\mathrm{s}}$ evolve according to

$$
\left.\frac{1}{a_{\mathrm{s}}} \frac{d a_{\mathrm{s}}}{d t}\right|_{v}=\left.\frac{d i_{\mathrm{s}}^{2}}{d t}\right|_{v}=-\left.\frac{2}{n_{\mathrm{s}}^{2} a_{\mathrm{s}}^{2} M_{\mathrm{s}}} \frac{d E}{d t}\right|_{v},
$$

where $(d E / d t)_{v}>0$ is the total rate of energy dissipation in all of the arcs.

The rate of energy dissipation may be written as

$$
\left.\frac{d E}{d t}\right|_{v} \approx \frac{M_{\mathrm{r}, \mathrm{tot}} v^{2}}{2 t_{\mathrm{c}}}
$$

where $v$ is random velocity of the ring particles, $t_{\mathrm{c}}$ is the interparticle collision time, and $M_{\mathrm{r}, \text { tot }}$ is the total mass of all the arcs. With the additional approximations $t_{\mathrm{c}}^{-1} \approx n$ and $v \approx n s$, where $s$ is the particle size, we obtain 


$$
\left.\frac{d i_{\mathrm{s}}^{2}}{d t}\right|_{v} \approx-\left(\frac{M_{\mathrm{r}, \mathrm{tot}}}{M_{\mathrm{s}}}\right)\left(\frac{s}{a}\right)^{2} n .
$$

Substitution of the nominal parameters, $M_{\mathrm{r}, \text { tot }} / M_{\mathrm{s}} \approx 10^{-4}$ and $s \approx 100 \mathrm{~cm}$, yields $\Delta i_{\mathrm{s}}^{2} \approx 4 \times 10^{-7}$ over a timespan of $5 \times 10^{9} \mathrm{yr}$, the age of the solar system. Hence the decay of $i_{\mathrm{s}}$ due to energy dissipation in the arcs is probably negligible.

\section{Satellite Eccentricity}

An alternate theory for the Neptune arcs might be based on the corotation resonances of a satellite that moves on an eccentric, equatorial orbit. With a few obvious changes, the dynamics of this theory would be analogous to the one that we have described. Current observations do not favor one theory over the other. Our preference for an inclined and circular satellite orbit is based entirely on calculations that indicate rapid tidal decay of eccentricity and negligible decay of inclination. The orbit of Triton is the prime example of this feature of tidal evolution (Goldreich and Soter 1966).

The tidal evolution of the satellite's orbital eccentricity is probably dominated by the tides raised in it by Neptune rather than by the tides it raises in the planet. Both types of tide act to damp $e_{\mathrm{s}}$; the latter since the satellite's orbital period ( $13.7 \mathrm{hr}$ at $a_{\mathrm{s}}=7.5 \times 10^{4} \mathrm{~km}$ ) is shorter than the planet's rotation period $(18.2 \pm 0.4 \mathrm{hr}$, see Harris 1984). The rate at which the eccentricity damps due to tidal dissipation in the satellite is given by (Goldreich and Soter 1966; Peale et al. 1980)

$$
\begin{aligned}
\frac{1}{e_{\mathrm{s}}} \frac{d e_{\mathrm{s}}}{d t}= & -3 \times 10^{-16} \mathrm{~s}^{-1}\left(\frac{10^{11} \mathrm{dyn} \mathrm{cm}^{-2}}{\mu}\right)\left(\frac{10^{2}}{Q}\right) \\
& \times\left(\frac{R_{\mathrm{s}}}{150 \mathrm{~km}}\right)^{4}\left(\frac{7.5 \times 10^{4} \mathrm{~km}}{a_{\mathrm{s}}}\right)^{13 / 2} .
\end{aligned}
$$

Here, $\mu$ and $Q$ are the tidal rigidity and quality factor, respectively. For the nominal parameter values the damping time is $10^{8} \mathrm{yr}$, much shorter than the age of the solar system.

The heating rate of the satellite implied by Eq. (30) is
$2 \times 10^{16} \mathrm{erg} \mathrm{s}^{-1}$, which suggests that its interior might have melted (Squyres et al. 1983 estimate that a heating rate of $10^{17} \mathrm{erg} \mathrm{s}^{-1}$ is sufficient to melt Enceladus, which has $R_{\mathrm{s}}$ $=250 \mathrm{~km}$ ). Substantial melting would reduce the effective rigidity of the satellite, thereby accelerating the rate at which $e_{\mathrm{s}}$ damps.

\section{CONCLUDING REMARKS}

We have outlined a theory that is consistent with the current fragmentary information about Neptune's arc rings. One point of concern is the requirement that the postulated satellite have a substantial orbital inclination since there is a clear tendency for the inner satellites of all planets to have equatorial orbits; Triton is the only known exception to this rule. We speculate that the initial orbital inclination of our hypothetical satellite resulted from the same event that was responsible for producing the unusual orbits of Nereid and Triton. The strikingly different morphologies of the Uranian and Neptunian ring systems are also attributed to this same event.

Support for our hypothesis could come from discovery of the postulated satellite and the determination of a nonzero inclination for its orbit. This crucial test should take place no later than the time at which the Hubble Space Telescope first looks at Neptune. However, it might come even earlier if a determined effort to detect new satellites is made using ground-based telescopes. Other crucial evidence would be the determination of different orbital radii for several arcs, if the radii were related as expected for different corotation resonances of a single satellite. Finally, we eagerly await the arrival, in good health, of Voyager 2 at Neptune in 1989.

We thank Phil Nicholson for discussions of the observations. This work was supported by grants from ATP Planétologie 1984 and 1985, by National Science Foundation grants AST-8412365 and AST-8521033, by NASA grant NGL-05-002-003, and by an operating grant from NSERC.

\section{REFERENCES}

Borderies, N., Goldreich, P., and Tremaine, S. (1982). Nature 299, 209. Borderies, N., Goldreich, P., and Tremaine, S. (1983). Astron. J. 88, 1560. Borderies, N., Goldreich, P., and Tremaine, S. (1984). Astrophys. J. 284, 429.

Borderies, N., Goldreich, P., and Tremaine, S. (1985). Icarus 63, 406.

Brouwer, D., and Clemence, G. M. (1961). Methods of Celestial Mechanics (Academic, New York).

Goldreich, P., and Soter, S. (1966). Icarus 5, 375.

Goldreich, P., and Tremaine, S. (1980). Astrophys. J. 241, 425.

Harris, A. W. (1984). In Uranus and Neptune, edited by J. T. Bergstralh (NASA, Washington, DC), p. 357.
Hubbard, W. B., Brahic, A., Sicardy, B., Elicer, L.-R., Roques, F., and Vilas, F. (1986). Nature 319, 636.

Lissauer, J. J. (1985). Nature 318, 544.

Nicholson, P. D. (1986). Private communication.

Peale, S. J., Cassen, P., and Reynolds, R. T. (1980). Icarus 43, 65.

Reitsema, H. J., Hubbard, W. B., Lebofsky, L. A., and Tholen, D. J. (1982). Science 215, 289.

Squyres, S. W., Reynolds, R. T., Cassen, P. M., and Peale, S. J. (1983). Icarus 53, 319.

Wasserman, L. H. (1986). Private communication. 